\title{
B7-dependent $T$-cell costimulation in mice lacking CD28 and CTLA4
}

\author{
Didier A. Mandelbrot, ${ }^{1}$ Mariette A. Oosterwegel, ${ }^{2}$ Koichi Shimizu, ${ }^{3}$ Akira Yamada,, 5 \\ Gordon J. Freeman, ${ }^{6}$ Richard N. Mitchell, ${ }^{1}$ Mohammed H. Sayegh, ${ }^{4}$ and Arlene H. Sharpe ${ }^{1}$ \\ ${ }^{1}$ Departments of Pathology, Brigham and Women's Hospital and Harvard Medical School, Boston, Massachusetts, USA \\ ${ }^{2}$ Department of Immunology, University Medical Center, Utrecht, The Netherlands \\ ${ }^{3}$ Department of Medicine, Brigham and Women's Hospital, Boston, Massachusetts, USA \\ ${ }^{4}$ Laboratory of Immunogenetics and Transplantation, Brigham and Women's Hospital and Harvard Medical School, \\ Boston, Massachusetts, USA \\ ${ }^{5}$ Transplantation Unit, Surgical Services, Massachusetts General Hospital and Harvard Medical School, Boston, \\ Massachusetts, USA \\ ${ }^{6}$ Department of Adult Oncology, Dana-Farber Cancer Institute, Boston, Massachusetts, USA \\ Address correspondence to: Arlene H. Sharpe, Immunology Research Division, Department of Pathology, \\ Brigham and Women's Hospital, Boston, Massachusetts 02115, USA. \\ Phone: (617) 278-0312; Fax: (617) 732-5795; E-mail: asharpe@rics.bwh.harvard.edu. \\ Didier A. Mandelbrot and Mariette A. Oosterwegel contributed equally to this work.
}

Received for publication November 3, 2000, and accepted in revised form January 18, 2001.

\begin{abstract}
To examine whether B7 costimulation can be mediated by a molecule on T cells that is neither CD28 nor CTLA4, we generated mice lacking both of these receptors. CD28/CTLA4 $4^{-/-}$mice resemble $C D 28^{-/-}$ mice in having decreased expression of T-cell activation markers in vivo and decreased T-cell proliferation in vitro, as compared with wild-type mice. Using multiple approaches, we find B7-dependent costimulation in $\mathrm{CD} 28 / \mathrm{CTLA4} 4^{-/-}$mice. The proliferation of $\mathrm{CD} 28 / \mathrm{CTLA4^{-/- }} \mathrm{T}$ cells is inhibited by CTLA4-Ig and by the use of antigen-presenting cells lacking both B7-1 and B7-2. CD28/CTLA4-/T-cell proliferation is increased by exposure to Chinese hamster ovary cells transfected with B7-1 or B7-2. Finally, administration of CTLA4-Ig to CD28/CTLA4-/- cardiac allograft recipients significantly prolongs graft survival. These data support the existence of an additional receptor for $\mathrm{B} 7 \mathrm{~mole}-$ cules that is neither CD28 nor CTLA4.
\end{abstract}

J. Clin. Invest. 107:881-887 (2001).

\section{Introduction}

The B7/CD28/CTLA4 pathway plays a critical role in the regulation of T-cell activation and has great potential as a therapeutic target in transplant rejection and autoimmunity. B7-1 (CD80) and B7-2 (CD86) provide a critical costimulatory signal to $\mathrm{T}$ cells by binding to CD28 (1) and inhibit T-cell activation by binding to CTLA4 (2).

Much has been learned about T-cell costimulation from the analysis of mice deficient in CD28 or B7-1/B7-2 molecules. Since B7-1 and B7-2 are the only known ligands for $\mathrm{CD} 28$ (3), it might have been expected that the defects in T-cell activation would be similar in these two mouse strains. However, dramatic differences are observed in the immune responses of the CD28-deficient $\left(\mathrm{CD} 28^{-/-}\right)$and $B 7-1 / B 7-2^{-/-}$mice. While $\mathrm{CD} 28^{-/-}$mice reject cardiac allografts almost as quickly as wild-type recipients (4), B7-1/B7-2-/- mice allow long-term survival (5). This result suggests that $\mathrm{B} 7$ costimulation of $T$ cells can occur through a receptor that is distinct from CD28. To determine whether there is B7-dependent costimulation of $\mathrm{T}$ cells in the absence of CD28 and CTLA4, we have generated a novel mouse strain lacking both CD28 and CTLA4.

Our in vitro studies demonstrate that either B7 blockade or the use of B7-deficient antigen-presenting cells (APCs) significantly reduces the proliferation of CD28/CTLA4 $4^{--}$T cells. Furthermore, B7-1 and B7-2 Chinese hamster ovary $(\mathrm{CHO})$ cell transfectants can stimulate proliferation of CD28/CTLA4 $4^{-/-} \mathrm{T}$ cells. In vivo, we find that administration of CTLA4-Ig to CD28/CTLA4 $4^{-/}$cardiac allograft recipients significantly prolongs graft survival. Together, these data support the existence of an additional receptor for $\mathrm{B} 7$ molecules that is neither CD28 nor CTLA4.

\section{Methods}

Generation of mice lacking CD28 and CTLA4. To generate CD28/CTLA4 ${ }^{-/}$mice we used a CD28 targeting vector to retarget a J1 embryonic stem (ES) cell clone (T5) that was heterozygous for the CTLA4 mutation and known to give rise to CTLA4-deficient mice exhibiting a lymphoproliferative phenotype (6). Because the T5 clone was neomycin resistant, the CD28 targeting vector carried the hygromycin-resistance gene as an alternative selection marker. The targeting vector (Figure 1a) was generated by replacing a $3-\mathrm{kb}$ region encompassing a portion of the IgV-like exon 2 and a portion of transmembrane exon 3 with hygromycin driven by the mouse phosphoglycerol kinase (PGK) promoter and linked to PGK poly(A) sequences. To select against random insertional 

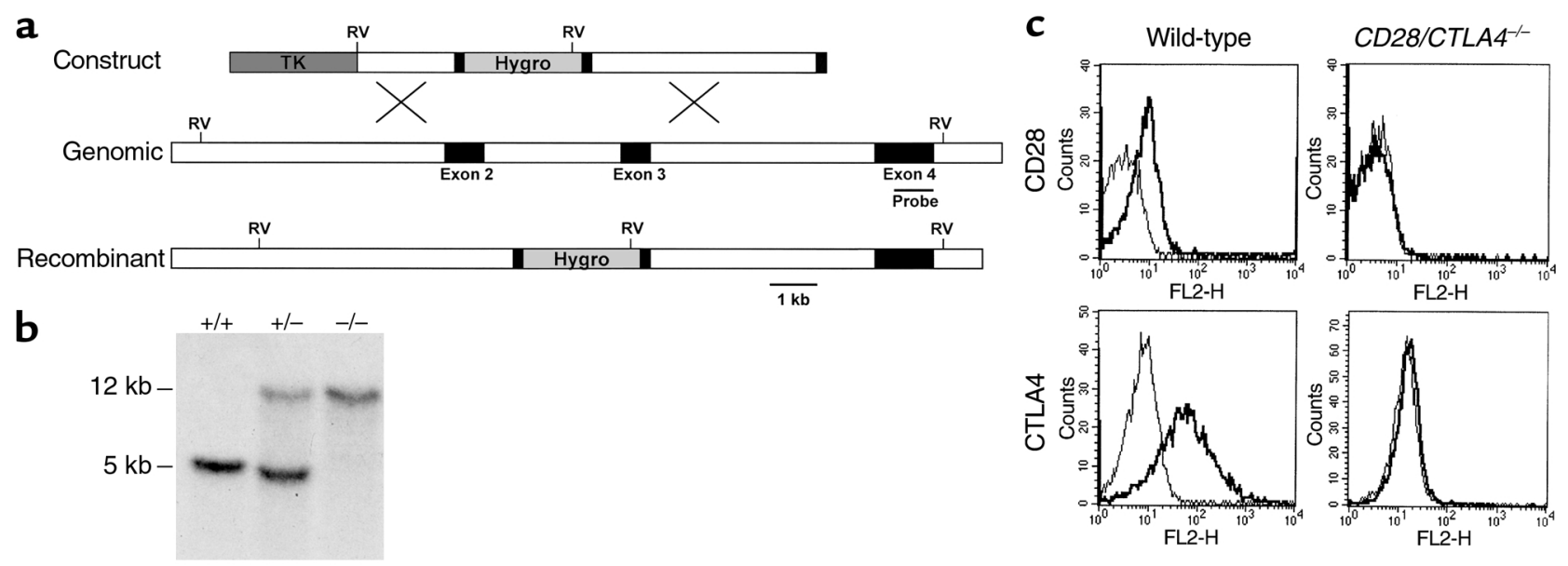

\begin{abstract}
Figure 1
Generation of mice lacking both CD28 and CTLA4. (a) Structure of the CD28 targeting construct. The hygromycin-resistance gene is flanked by small parts of exon 2 and 3 . The thymidine kinase gene (TK) lies external to the $5^{\prime}$ genomic fragment. The intron-exon organization of the genomic clone is shown. Exons coding for the extracellular, transmembrane, and intracellular domain of CD28 are depicted as filled rectangles. The 0.9-kb cDNA probe used for Southern blot analysis is indicated as a thin bar and is located in exon 4, external to the genomic DNA fragment used in the targeting construct. Gene targeting in ES cells by homologous recombination results in the replacement of part of exon 2, the intron between exon 2 and 3, and part of exon 3 by the hygromycin gene. Southern blot analysis uses the acquisition of an additional EcoRV site in the hygromycin gene (shown in bold) to distinguish genomic and targeted DNA. (b) Southern blot analysis of the CD28 locus. Tail DNA from wild-type (+/+), heterozygotes (+/-), and CD28/CTLA4 double-knockout (-/-) littermates were digested with EcoRV and hybridized with the probe indicated in Figure 1a, demonstrating the two bands indicative of the endogenous (12 kb) or targeted (5 kb) configuration. (c) Analysis of CD28 and CTLA4 expression. Cell-surface staining of CD28 is assayed on naive CD4 T cells; CTLA4 is stained intracellularly in activated CD4 cells. Thick lines depict staining with anti-CD28 or anti-CTLA4; thin lines depict staining with the isotype-matched hamster IgG control Ab.
\end{abstract}

events, a thymidine kinase gene (TK) under the regulation of the MC1 promoter was incorporated at the $5^{\prime}$ end of the targeting vector. The targeting vector contains 2 $\mathrm{kb}$ of homology upstream and $4 \mathrm{~kb}$ of homology downstream of the PGK-hygro gene. After electroporation, hygromycin- and FIAU-resistant ES cells were selected and screened by Southern blot analysis. Genomic DNA was digested with EcoRV and hybridized with a $0.9-\mathrm{kb}$ probe outside the construct. A DNA fragment of $12 \mathrm{~kb}$ corresponded to the wild-type allele, and a fragment of $5 \mathrm{~kb}$ indicated homologous recombination at the CD28 locus. Single integration events were scored using a probe derived from the hygromycin gene. Three distinct ES cell lines heterozygous for the CD28 mutation were microinjected into blastocysts and gave rise to germline transmission of the CD28 mutation. Using the neomycin and hygromycin drug-resistance genes as markers for the individual targeting events, we selected for progeny in which these markers cosegregated, indicating both mutations were on the same chromosome. Offspring carrying both the neomycin- and hygromycinresistance genes were identified and interbred. Progeny were determined by Southern blot analysis and PCR of tail DNA. The PCR primers used were: $\mathrm{mCD} 28$ 5'AACAAGATTTTGGTAAAGCAG; mCD28 3' GAACTCAATTTTGCAGAAGTA; Hygro 5' GACCTGCCTGAAACCGAACT; Hygro 3' ACCAATGCGGAGCATATACG; mCTLA4 $5^{\prime}$ TGGTGTTGGCTAGCAGCCATG; mCTLA4 $3^{\prime}$ TTGGATGGTGAGGTTCACTC; Neo 5' ATTGAACAAGATGGATTGCAC; Neo 3' CGTCCAGATCATCCTGATC.
Mice. Wild-type and $\mathrm{CD} 28^{-/-}$mice on the $\mathrm{BALB} / \mathrm{c}$ and C57BL/6 (B6) backgrounds were obtained from the Jackson Laboratory (Bar Harbor, Maine, USA). CD28/CTLA4 $4^{-/}$mice were used for flow cytometry and proliferation assays after back-crossing for at least five generations onto the $\mathrm{BALB} / \mathrm{c}$ background. The $\mathrm{BALB} / \mathrm{c} B 7-1^{-/-}, B 7-2^{-/-}$, and $B 7-1 / B 7-2^{-/-}$mice (7) were back-crossed for ten generations. Brigham and Women's Hospital and Harvard Medical School are accredited by the American Association of Accreditation of Laboratory Animal Care (AALAC), and mice were cared for in accordance with institutional guidelines in a pathogen-free animal facility.

Flow cytometry. CD28 expression was determined by staining freshly isolated lymph node cells with Ab's to CD4 and CD28 (or isotype control). To determine CTLA4 expression, $\mathrm{CD} 4^{+} \mathrm{T}$ cells were purified from peripheral lymph nodes using magnetic beads (MACS) from Miltenyi Biotec (Auburn, California, USA) and stimulated with anti-CD3 and APCs for 3 days. Cells were stained for $\mathrm{CD} 4$, and intracellular CTLA4 expression was determined after treating cells with $0.5 \%$ saponin in $0.5 \%$ BSA. To assess T-cell activation, freshly isolated spleen and lymph node cells were stained for CD4 and the activation markers CD69 or CD62L. All $\mathrm{Ab}$ 's were directly conjugated and were obtained from PharMingen (San Diego, California, USA).

Proliferation assays. All in vitro assays were performed using mice on the BALB/c background. $\mathrm{CD}^{+} \mathrm{T}$ cells were purified by MACS, with purity greater than $95 \%$ 
confirmed by flow cytometry. $T$ cells were plated in flatbottom wells at $5 \times 10^{4}$ cells/well in RPMI medium as described previously (3). APCs from T-depleted splenocytes (previously described; ref. 8) were stimulated overnight with $5 \mu \mathrm{g} / \mathrm{ml}$ of the anti-CD40 Ab 3/23, then treated with mitomycin C $(50 \mu \mathrm{g} / \mathrm{ml}$ for 40 minutes at $37^{\circ} \mathrm{C}$ ) and plated at $5 \times 10^{5}$ cells/well. B7 transfectants of CHO cells $(9,10)$ were mitomycin C treated $(50$ $\mu \mathrm{g} / \mathrm{ml}$ overnight) and plated at $2 \times 10^{4}$ cells/well. The hybridoma for the 145-2C11 Ab to CD3 was obtained from the American Type Tissue Culture (Manassas, Virginia, USA), and $\mathrm{Ab}$ was used either as a high-titer supernatant or purified. Purified CTLA4-Ig was kindly provided by Genetics Institute (Cambridge, Massachusetts, USA). Secondary stimulations were performed by culturing freshly isolated $\mathrm{CD}^{+}$cells with APCs and 1:1000 dilution of anti-CD3 supernatant for 4 days, resting overnight in $20 \mathrm{U} / \mathrm{ml} \mathrm{IL-2,} \mathrm{and} \mathrm{restimulating}$ equal T-cell numbers with APCs and the indicated concentration of anti-CD3 for 3 days.

Cytokine measurement. Supernatants from T-cell cultures were analyzed for IL-2, IL-4, IL-10, and IFN- $\gamma$ by sandwich ELISA using $\mathrm{Ab}$ pairs and standards purchased from PharMingen, according to the manufacturer's instructions.

Heart transplantation. Allografts from male donors were placed in male recipients as described previously (11). Graft function was assessed daily by palpation, with rejection defined as the absence of detectable beating. Allografts failing or graft recipients dying within 48 hours of surgery were considered technical failures and were excluded from the analysis. Donor hearts from wild-type C57BL/6 (H-2 $\left.{ }^{\text {b }}\right)$ mice were transplanted to either $\mathrm{BALB} / \mathrm{c}\left(\mathrm{H}-2^{\mathrm{d}}\right)$ wild-type, $\mathrm{CD} 28^{-/-}$, or CD28/CTLA4 $4^{-/-}$mice. Donor hearts from wild-type BALB/c mice were transplanted to wild-type recipients on either a $\mathrm{B} 6$ background or a mixed 129/B6 background or CD28/CTLA4-/- recipients on a mixed 129/B6 background.

\section{Results}

Generation of mice lacking CD28 and CTLA4. Because CD28 and CTLA4 are closely linked on mouse chromosome 1, we chose to target the CD28 gene in ES clones heterozygous for the CTLA4 mutation, which were used to generate the CTLA4-deficient mice that we have characterized previously (6). Our targeting vector (Figure 1a) replaced sequences encoding the IgVlike exon 2 and a portion of the transmembrane exon 3 of CD28 with the hygromycin-resistance gene, which should effectively destroy CD28 binding to B7-1 and B7-2. Germline-transmitting progeny from targeted ES cell clones were bred to wild-type BALB/c or C57BL/ 6 mice. Progeny heterozygous for both the CD28 and CTLA4 mutation were interbred and gave rise to viable mice carrying both mutations with the expected frequency, as determined by Southern blot analysis (Figure 1b). The absence of CD28 and CTLA4 expression was confirmed by flow cytometry (Figure 1c).
CD28/CTLA4-/- mice do not exbibit the lymphoproliferative phenotype of CTLA4 $4^{--}$mice. CD28/CTLA4 $4^{-/-}$mice have a life span similar to wild-type littermates, in contrast to CTLA4 $4^{-/-}$mice, which die at 3 weeks of age $(6,12,13)$. In addition, the CD28/CTLA4 $4^{--}$mice do not develop the splenomegaly, lymphadenopathy, and lymphocytic infiltrates observed in the CTLA4 ${ }^{-/-}$mice. Also unlike the CTLA4 $4^{-/}$mice, $\mathrm{T}$ cells in CD28/CTLA4 ${ }^{-/-}$mice are not activated, as determined by flow cytometry. In freshly isolated lymph node or spleen cells, the proportion of $\mathrm{CD}^{+} \mathrm{T}$ cells from $\mathrm{CD} 28^{-/-}$and $\mathrm{CD} 28 / \mathrm{CTLA} 4^{-/}$ mice expressing the activation marker CD69 is similar and is approximately $50 \%$ lower than in T cells from wild-type mice (Figure 2). T cells from $\mathrm{CD}_{28^{-/-}}$and CD28/CTLA4 $4^{-/}$mice also show similar increases in CD62L expression, reflecting decreased activation as compared with wild-type mice (data not shown). In contrast, freshly isolated lymph node $\mathrm{T}$ cells from CTLA4 $4^{-/}$mice show dramatic increases in markers of activation as compared with wild-type mice $(3,6)$. Staining of CD28/CTLA4 ${ }^{-/}$cells from thymus, spleen, and lymph node for CD4, CD8, and B220 suggest that T-cell and B-cell development is unchanged from wildtype (data not shown).

In vitro proliferation of $\mathrm{CD} 28 / \mathrm{CTLA4} 4^{-/} \mathrm{T}$ cells is reduced and is associated with production of Th1 cytokines. Primary stimulation of $\mathrm{CD}^{+} \mathrm{T}$ cells from wild-type, $\mathrm{CD} 28^{--}$
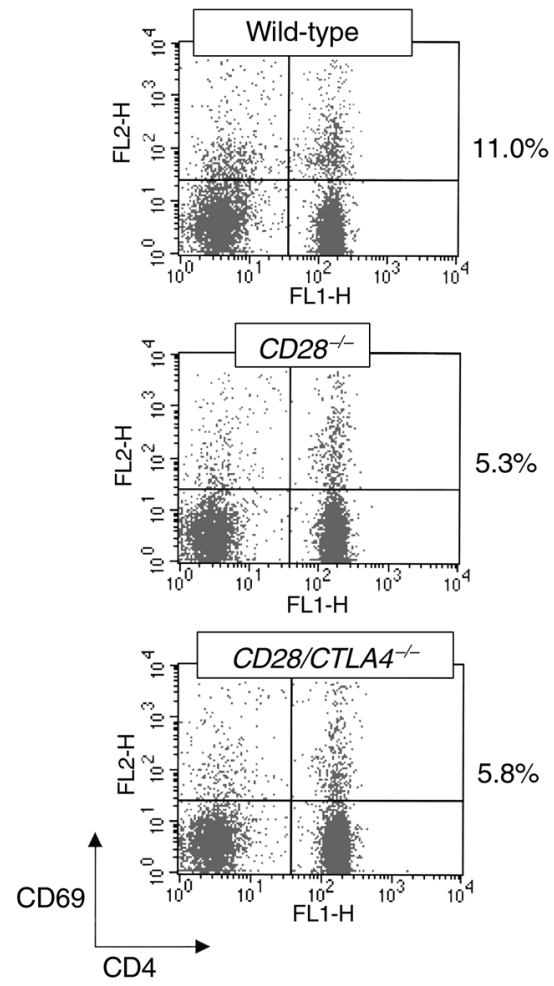

Figure 2

T-cell activation in naive mice. Freshly isolated lymph node cells from the indicated strains were stained for CD4 and CD69 and analyzed by flow cytometry. The percentages to the right of the dot plots are the proportion of $\mathrm{CD} 4^{+}$cells that are $\mathrm{CD} 69^{+}$. This experiment is representative of five mice of each strain. 

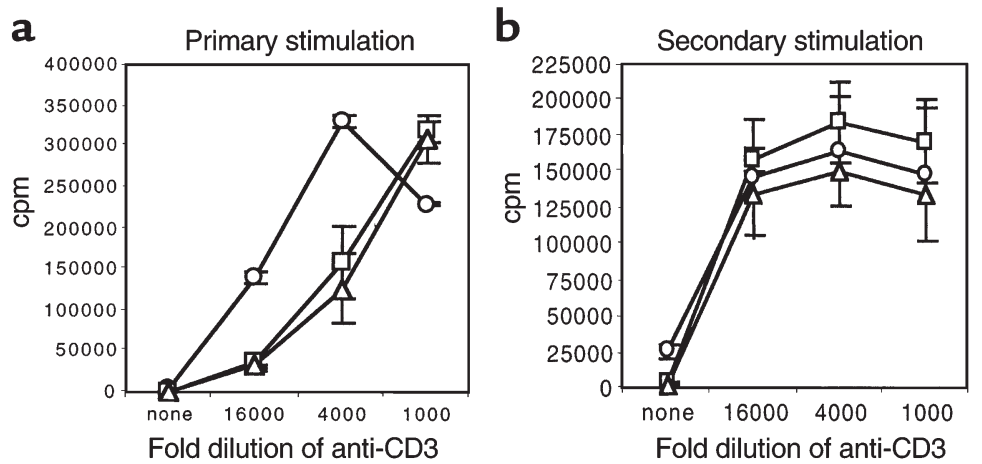

Figure 3

Proliferation of $\mathrm{CD} 4^{+} \mathrm{T}$ cells from wild-type (WT), CD28-/-, and CD28/CTLA4 $4^{-/-}$mice in the presence of wild-type APCs. (a) Primary stimulation of CD4 $4^{+}$ $T$ cells with the indicated dilutions of anti-CD3. Proliferation on day 3 is shown. Data are representative of five experiments. (b) Secondary stimulation of $\mathrm{CD}^{+} \mathrm{T}$ cells with the indicated dilutions of anti-CD3. Proliferation on day 2 is shown. Data are representative of three experiments.

and CD28/CTLA4 $4^{-/}$mice using wild-type APCs and anti-CD3 is shown in Figure 3a. $\mathrm{CD}^{+}$cells from the $\mathrm{CD} 28^{-/-}$and $\mathrm{CD} 28 / \mathrm{CTLA4}^{-/-}$mice proliferate less than wild-type $\mathrm{CD}^{+}$cells, but no significant difference is observed between $\mathrm{CD} 28^{-/-}$and $\mathrm{CD} 28 / \mathrm{CTLA4}^{-/-}$cells. Upon secondary stimulation, no significant differences in proliferation of $\mathrm{CD}^{+} \mathrm{T}$ cells from the three strains were observed (Figure 3b). Culture supernatants from both primary and secondary stimulations were assayed for IL-2, IL-4, IL-10, and IFN- $\gamma$ using ELISA. In primary stimulation cultures, IL-2 production was reduced by approximately $50 \%$ in the $\mathrm{CD} 28^{-/-}$and $\mathrm{CD} 28 / \mathrm{CTLA}^{-/-}$ mice, as compared with wild-type, and other cytokine levels were relatively low in all mice (data not shown). In the secondary stimulation, supernatants from CD28/CTLA4 $4^{--}$T cells showed a deviation to a Th1 pattern of cytokines, with increased production of IFN- $\gamma$, and decreased IL-4 and IL-10, as compared with wildtype mice (Figure 4). These findings are consistent with previous studies demonstrating the role of $\mathrm{CD} 28$ in the production of IL-2 (14) and the importance of CD28 for Th2 differentiation (15).

CD28/CTLA4 $4^{--}$T-cell proliferation is dependent on B7 molecules. To determine whether the activation of T cells lacking both CD28 and CTLA4 is dependent on B7 molecules, we used two complementary approaches. First, we compared proliferative responses of BALB/c wild-type, $C D 28^{-/-}$, and CD28/CTLA4-/- T cells to anti$\mathrm{CD} 3$ in the presence of syngeneic APCs from either wild-type, $B 7-1^{-/-}, B 7-2^{-/-}$, or $B 7-1 / B 7-2^{-/-}$mice. As expected, proliferation of wild-type $\mathrm{CD} 4^{+}$cells in the presence of wild-type APCs is significantly reduced by the addition of CTLA4-Ig, which blocks B7-1 and B7-2 (Figure 5). Strikingly, proliferation of $\mathrm{CD}^{+} \mathrm{T}$ cells from $\mathrm{CD} 28 / \mathrm{CTLA} 4^{-/-}$mice, as well as $\mathrm{CD} 28^{-/-}$mice, is also inhibited by CTLA4-Ig. Similarly, proliferation of $\mathrm{T}$ cells from all three responder strains is greatly reduced in the presence of $B 7-1 / B 7-2^{--}$APCs, but is not impaired with $B 7-1^{-/-}$or $B 7-2^{-/-}$APCs (data not shown). In addition, inhibition of $\mathrm{B} 7$ costimulation by using either CTLA4-Ig or B7-1/B7-2-/- APCs completely blocked production of IL-2, IL-4, IL-10, and IFN- $\gamma$ in the primary cultures of wild-type, $\mathrm{CD} 28^{-/-}$, and CD28/CTLA4 $4^{-1-}$ T cells (data not shown). These data demonstrate that $\mathrm{B} 7$-dependent costimulation is present in both $\mathrm{CD} 28^{-/-}$and CD28/CTLA4 $4^{-/-} \mathrm{T}$ cells.

We used $\mathrm{CHO}$ cell transfectants to further examine the $\mathrm{B} 7$ dependence of $\mathrm{CD} 28 / \mathrm{CTLA}^{-/-} \mathrm{T}$-cell proliferation. $\mathrm{CHO}$ cells were transfected with I-A $\mathrm{A}^{\mathrm{d}}$ or with I-A ${ }^{\mathrm{d}}$ plus B7-1, B7-2, or B7-1/B7-2. As shown in Figure 6, CHO cells expressing either $\mathrm{B} 7-1$ or $\mathrm{B} 7-2$ costimulate the proliferation of $\mathrm{CD} 28 / \mathrm{CTLA4}^{-/-}, \mathrm{CD} 28^{-/-}$, and wildtype $\mathrm{CD}^{+} \mathrm{T}$ cells. These results suggest that either B7-1 or B7-2 can stimulate $T$ cells in vitro through a receptor that is neither CD28 nor CTLA4.

Rejection of cardiac allografts by CD28/CTLA4 $4^{-/-}$mice is dependent on B7. To determine whether CD28/CTLA4-/$\mathrm{T}$ cells are dependent on B7 costimulation in vivo as well as in vitro, we used a model of heterotopic cardiac transplantation between fully MHC mismatched
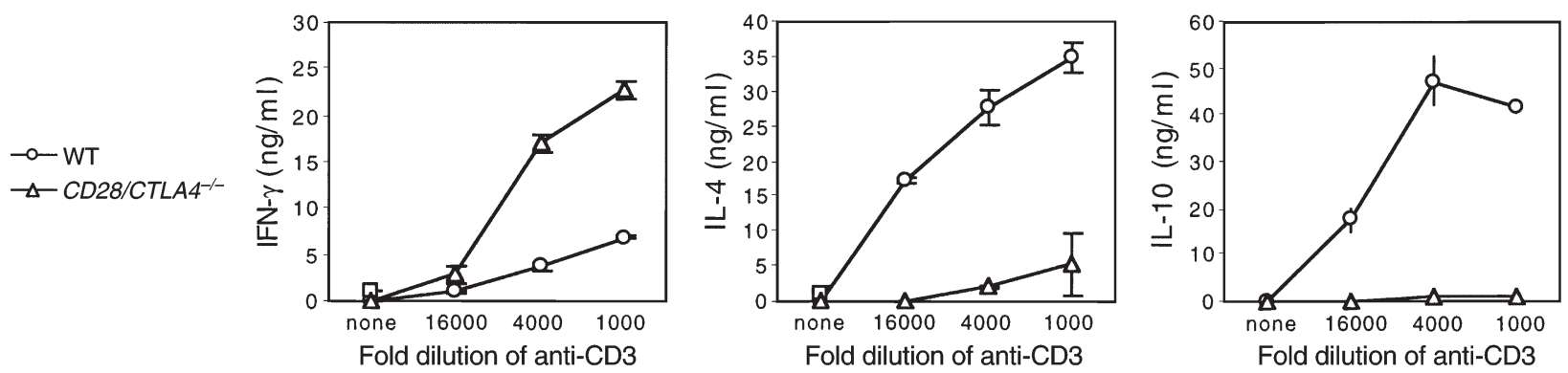

Figure 4

Cytokine production on day 2 after secondary stimulation of wild-type and CD28/CTLA4 ${ }^{-/-} C D 4^{+} T$ cells with the indicated dilutions of anti-CD3. Data are representative of four experiments. 

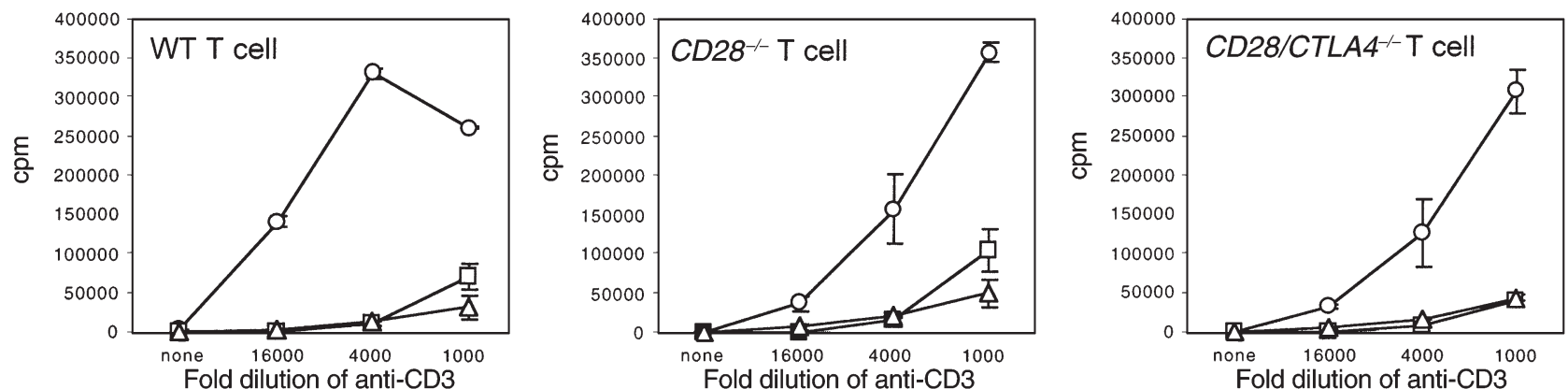

$\multimap-W T \quad \rightarrow-W T+C T L A 4-\lg \quad-\triangle-B 7-1 / B 7-2^{--}$

\section{Figure 5}

Proliferation of $\mathrm{CD}^{+} \mathrm{T}$ cells from wild-type, $\mathrm{CD} 28^{-/-}$, and $\mathrm{CD} 28 / \mathrm{CTLA4} 4^{-/-}$mice in the presence of syngeneic APCs. T cells from the indicated strains were stimulated in the presence of wild-type APCs with or without CTLA4-Ig or in the presence of B7-deficient APCs. Proliferation on day 3 is shown. Data are representative of five experiments.

mouse strains. As shown in Figure 7a, when wild-type $\mathrm{BALB} / \mathrm{c}\left(\mathrm{H}-2^{\mathrm{d}}\right)$ hearts were transplanted to $\mathrm{B} 6\left(\mathrm{H}-2^{\mathrm{b}}\right)$ wild-type or CD28/CTLA4 ${ }^{-/-}$recipients, allograft survival was slightly prolonged in CD28/CTLA4 $4^{-/}$recipients (mean survival time [MST] 15.5 days, $n=10$ ) as compared with wild-type recipients (MST 9.4 days, $n=5$ ), but this did not reach statistical significance. Importantly, CTLA4-Ig treatment prolonged graft survival in CD28/CTLA4-/- recipients significantly (MST 31.5 days, $n=11 ; P \leq 0.05)$ compared with nontreated CD28/CTLA4 $4^{-/}$recipients, albeit less than occurred in CTLA4-Ig-treated wild-type cardiac allograft recipients (MST $\geq 90$ days, $n=3 ; P \leq 0.02$ ). These results demonstrate B7-dependent costimulation in CD28/CTLA4 $4^{-/}$ mice in vivo. The finding that CTLA4-Ig was less effective in the CD28/CTLA4-/- as compared with wild-type recipients is consistent with the our previous observations (16) showing that an intact CTLA4 signaling pathway is required for induction of long-term allograft survival by CTLA4-Ig.

Allograft survival by $\mathrm{CD} 28^{-/-}$and $C D 28 / C T L A 4^{-/-}$recipients. To compare cardiac allograft survival in mice lacking CD28 with mice lacking CD28 and CTLA4, we analyzed the survival of allografts from wild-type C57BL/6 $\left(\mathrm{H}-2^{\mathrm{b}}\right)$ mice transplanted into BALB $/ \mathrm{c}\left(\mathrm{H}-2^{\mathrm{d}}\right)$ wild-type, $\mathrm{CD} 28^{-/-}$, and $\mathrm{CD} 28 / \mathrm{CTLA4} 4^{-/-}$recipients (Figure 7b). Allograft survival in BALB/c CD28 $8^{--}$ recipients was significantly prolonged (MST 22.6 days, $n=5 ; P \leq 0.001)$ as compared with BALB/c wildtype recipients (MST 9.0 days, $n=7$ ), but the $\mathrm{CD}_{28^{-/-}}$ recipients did reject the cardiac allografts, consistent with previous studies (4). Allograft survival was significantly shorter in BALB/c CD28/CTLA4 ${ }^{-/-}$recipients (MST 10.3 days, $n=6 ; P \leq 0.002$ ) as compared with $\mathrm{CD} 28^{-/-}$recipients and similar to wild-type $\mathrm{BALB} / \mathrm{c}$ recipients (MST 9.0 days, $n=7$ ). The more rapid rejection of cardiac allografts in CD28/CTLA4 $4^{-/}$ as compared with $C D 28^{-/-}$recipients is consistent with the absence of negative signaling through CTLA4 and is comparable to the accelerated rejection observed when a blocking anti-CTLA4 $\mathrm{mAb}$ was administered to $\mathrm{CD} 28^{-/-}$allograft recipients (4).

\section{Discussion}

In this report, we have used a newly generated mouse strain lacking CD28 and CTLA4 to examine B7-
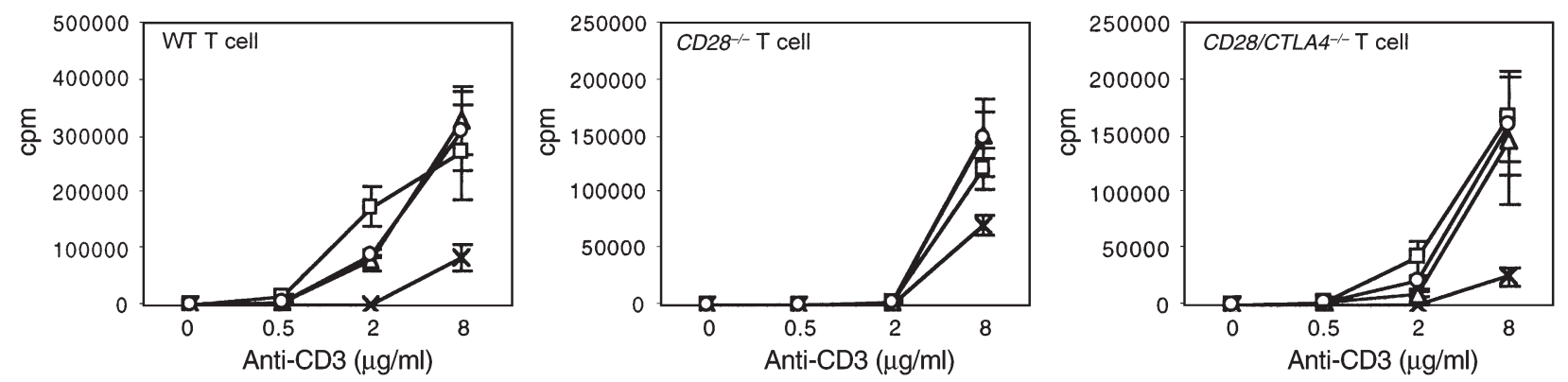

APC

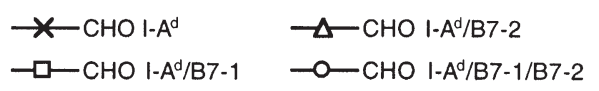

Figure 6

Proliferation of $\mathrm{CD} 4{ }^{+} \mathrm{T}$ cells from wild-type, $\mathrm{CD} 28^{-/-}$, and CD28/CTLA4 $4^{-/-}$mice in the presence of the indicated concentration of anti-CD3 and $\mathrm{CHO}$ cells transfected with I-A $A^{d}$ or I- $A^{d}$ plus the indicated B7 molecules. Proliferation on day 3 is shown. Data are representative of three experiments. 

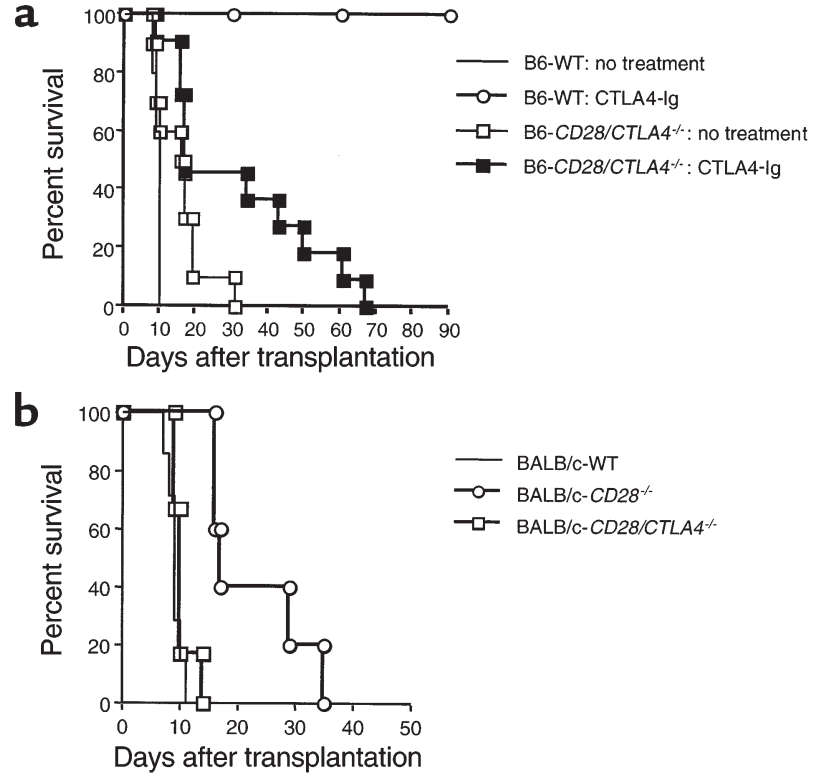

Figure 7

Cardiac transplantation studies. (a) BALB/c $\left(\mathrm{H}-2^{\mathrm{d}}\right)$ hearts were transplanted to $\mathrm{B} 6\left(\mathrm{H}-2^{\mathrm{b}}\right)$ wild-type or $\mathrm{CD} 28 / \mathrm{CTLA4} 4^{-/-}$recipients with or without CTLA4-Ig treatment. Graft survival was slightly prolonged in CD28/CTLA4 ${ }^{-/-}$recipients (MST 15.5 days, $n=10$ ), but there was no statistical significance compared with wild-type recipients (MST 9.4 days, $n=5 ; P=\mathrm{NS})$. CTLA4-Ig treatment in wild-type recipients prolonged graft survival significantly (MST $>90$ days, $n=3 ; P<0.02$ ) compared with nontreated wild-type recipients. CTLA4-Ig treatment also prolonged graft survival in CD28/CTLA4 ${ }^{-/-}$recipients significantly (MST 31.5 days, $n=11 ; P<0.05$ ) compared with nontreated CD28/CTLA4 ${ }^{-1-}$ recipients; however, that prolongation was much less than in wild-type recipients $(P<0.005)$. (b) Vascularized B6 $\left(\mathrm{H}-2^{\mathrm{b}}\right)$ hearts were transplanted to BALB/c $\left(\mathrm{H}-2^{\mathrm{d}}\right)$ wild-type, $\mathrm{CD} 28^{-/-}$, or CD28/CTLA4 $4^{-/-}$recipients. The graft survival in CD28 $8^{-/-}$recipients was prolonged (MST 22.6 days, $n=5$ ) compared with both wild-type (MST 9.0 days, $n=7 ; P<0.001$ ) and CD28/CTLA4 $4^{-/}$recipients (MST 10.3 days, $n=6 ; P<0.002)$. CD28/CTLA4-/- recipients rejected $\mathrm{B} 6$ heart grafts at the same tempo as wild-type recipients.

dependent costimulation in the absence of the two known receptors for $\mathrm{B} 7$. The phenotype of this mouse more closely resembles the $\mathrm{CD} 28^{-/-}$mouse (17) than the CTLA4 $4^{--}$mouse. In particular, we have no evidence of the lymphoproliferative disease seen in $C T L A 4^{-/}$mice. $\mathrm{T}$ cells from the $\mathrm{CD} 28^{--}$and $\mathrm{CD} 28 / \mathrm{CTLA4^{-/ }}$ mice show similarly impaired activation ex vivo (Figure 2 ) and reduced $\mathrm{CD}^{+} \mathrm{T}$-cell proliferative responses in vitro (Figure 3). These findings are consistent with the requirement for $\mathrm{CD} 28$ to produce the uncontrolled lymphocyte proliferation of the CTLA4 $4^{-/-}$mouse. Strikingly, we find that $\mathrm{T}$ cells lacking both CD28 and CTLA4 exhibit B7-dependent proliferation, suggesting the existence of a third receptor for B7-1 and B7-2.

The putative third receptor for $\mathrm{B} 7$ provides a costimulatory signal to $\mathrm{T}$ cells that is strong enough to mediate allograft rejection, although it does not lead to a lymphoproliferative phenotype in vivo. There are several potential mechanisms by which the new $\mathrm{B} 7$ receptor may not lead to the lymphoproliferative phenotype that has been seen in CTLA4-/- mice in vivo $(6,12)$. First, it is possible that the new receptor is inducible and thus gets upregulated in response to immune stimuli (such as allograft rejection, an autoimmune response, or response to a pathogen). Second, other negative regulatory pathways, such as the PD-1:PD-L pathway (18), may prevent T-cell activation by the third receptor in the CD28/CTLA4 ${ }^{-/-}$mouse. Recent studies indicate that that the PD-L:PD-1 pathway can inhibit T-cell receptor-mediated (TCR-mediated) proliferation and cytokine production by $\mathrm{CD}^{+} \mathrm{T}$ cells. PD-1:PD-L interactions also can antagonize TCR/CD28 signals, depending on the strength of the TCR signal. The PD-1:PD-L pathway could similarly inhibit costimulation by the new $\mathrm{B} 7$ receptor.

Previous studies failed to demonstrate B7-dependent costimulation in $\mathrm{CD} 28^{-/-} \mathrm{T}$ cells (19). However, using three different in vitro approaches, we demonstrate that $\mathrm{T}$ cells from both the $\mathrm{CD} 28^{-/-}$and CD28/CTLA4 $4^{-/}$mice can be costimulated by B7 (Figures 5 and 6). Blockade of B7 using CTLA4-Ig or using B7-1/B7-2-/- APCs decreases CD4 ${ }^{+} \mathrm{T}$-cell proliferation in both mouse strains. In addition, $\mathrm{CHO}$ cells transfected with B7-1 or B7-2 increase the proliferation of both $\mathrm{CD} 28^{-/-}$and $C D 28 / C T L A 4^{-/-} \mathrm{T}$ cells. It is possible that previous studies in $\mathrm{CD}_{28^{-/-}}$mice did not elicit B7dependent stimulatory signals because the mouse strains or experimental conditions favored B7-CTLA4 interactions. In fact, we found that the effect of B7 blockade or $B 7-1 / B 7-2^{-1-}$ APCs in vitro was less dramatic when $T$ cells were from mice on a C57BL/6, rather than a BALB/c background. Furthermore, we demonstrated the effect of $B 7$ costimulation using $\mathrm{CHO}$ transfectants expressing high levels of B7 or APCs that were preincubated with anti-CD40 so that B7 is upregulated from the start of the T-cell cultures.

We have demonstrated previously that cardiac allograft survival depends only on the expression of B7 molecules in graft recipients and that B7 expression on donor cells does not affect survival (3). Therefore, our finding that B7-1/B7-2 molecules can costimulate $\mathrm{T}$ cells through a third receptor potentially explains the difference in allograft survival in $B 7-1 / B 7-2^{-/-}$versus $C D 28^{-/-}$recipients. Only in the $B 7-1 / B 7-2^{-/-}$recipient is B7-dependent costimulation completely absent. In contrast, in the $\mathrm{CD} 28^{-/-}$mouse, B7 can costimulate through a third receptor. Similarly, B7 costimulation in CD28/CTLA4 $4^{-/}$mice can mediate allograft rejection, and B7 blockade prolongs graft survival. Our data also provide an alternative explanation for previous studies in $\mathrm{CD} 28^{-/-}$mice that suggested that B7dependent costimulation occurs through CTLA4 (20), since this costimulation may, in fact, occur through a third receptor for B7.

The relative impact of B7 interactions with CTLA4 versus the third receptor appears to depend significantly on experimental conditions. For example, CTLA4-Ig blockade of $\mathrm{B} 7$ in $\mathrm{CD} 28^{-/-}$mice decreases $\mathrm{CD}^{+} \mathrm{T}$ cell proliferation in our in vitro system, but it accelerates 
cardiac allograft rejection in $C D 28^{-/-}$recipients (4). In CD28/CTLA4 ${ }^{-/}$mice, CTLA4-Ig blocks a stimulatory signal, because these mice lack CTLA4 molecules to compete for $\mathrm{B} 7$ binding. It may be that the avidity of $\mathrm{B} 7$ molecules is greater for CTLA4 than for the third receptor for B7. A higher avidity of B7 molecules for CTLA4 would explain why B7 blockade with CTLA4-Ig reduces allograft survival in $\mathrm{CD} 28^{-/-}$recipients, while prolonging survival in $C D 28 / C T L A 4^{-/-}$recipients.

Our results suggest that there are still more members of the growing B7 and CD28 families of costimulatory molecules (21). The recently reported ICOS (inducible costimulator) molecule (22), which is homologous to $\mathrm{CD} 28$, was shown to bind the B7h molecule (also called B7RP-1), which is homologous to B7-1 and B7-2 (23), and stimulate T-cell activation. However, the ICOS-B7h pathway does not explain the costimulation from $\mathrm{B} 7-1 / \mathrm{B} 7-2$ produced in the absence of CD28 and CTLA4, since B7-1 and B7-2 do not bind ICOS (24). The presence of a third B7 receptor on $\mathrm{T}$ cells demonstrates an additional level of complexity in T-cell costimulation and has fundamental implications for the manipulation of costimulatory pathways for therapeutic purposes in immune-mediated diseases. In that regard, it is important to understand these complex interactions not only in rodent models but also in primates and humans for the purpose of developing novel immunomodulatory strategies that can be successfully translated to the clinic.

\section{Acknowledgments}

We wish to thank Sumi Scott, Baolin Chang, Frans Hofhuis, and Sandra Jainandunsing for expert technical assistance; Scott Boyd and Andy Chen for their contributions to making the targeting construct; Peter Libby for his support of the transplant experiments; and Mary Collins of Genetics Institute for the gift of CTLA4-Ig. This work was supported by grants from the American Heart Association (D.A. Mandelbrot), the Human Frontier Science Program (M.A. Oosterwegel), and NIH grants AI-39671, CA-75174, AI-41584 (G.J. Freeman), HL 43364 (Peter Libby and R.N. Mitchell), AI-34965 and AI-41521 (M.H. Sayegh), and AI-41584 and AI-38310 (A.H. Sharpe).

1. McAdam, A.J., Schweitzer, A.N., and Sharpe, A.H. 1998. The role of B7 co-stimulation in activation and differentiation of CD4+ and CD8+ T cells. Immunol. Rev. 165:231-247.

2. Oosterwegel, M.A., Greenwald, R.J., Mandelbrot, D.A., Lorsbach, R.B., and Sharpe, A.H. 1999. CTLA-4 and T cell activation. Curr. Opin. Immunol. 11:294-300.

3. Mandelbrot, D.A., McAdam, A.J., and Sharpe, A.H. 1999. B7-1 or B7-2 is required to produce the lymphoproliferative phenotype in mice lacking cytotoxic T lymphocyte-associated antigen 4 (CTLA-4). J. Exp. Med. 189:435-440.

4. Lin, H., et al. 1998. Cytotoxic T lymphocyte antigen 4 (CTLA4) blockade accelerates the acute rejection of cardiac allografts in CD28-deficient mice: CTLA4 can function independently of CD28. J. Exp. Med. 188:199-204.

5. Mandelbrot, D.A., et al. 1999. Expression of B7 molecules in recipient, not donor, mice determines the survival of cardiac allografts. J. Immunol. 163:3753-3757.

6. Tivol, E., et al. 1995. Loss of CTLA-4 leads to massive lymphoproliferation and fatal multiorgan destruction, revealing a critical negative regulatory role of CTLA-4. Immunity. 3:541-547.

7. Borriello, F., et al. 1997. B7-1 and B7-2 have overlapping, critical roles in immunoglobulin class switching and germinal center formation. Immunity. 6:303-313.

8. Oosterwegel, M.A., et al. 1999. The role of CTLA-4 in regulating Th2 differentiation. J. Immunol. 163:2634-2639.

9. Galvin, F., et al. 1992. Murine B7 antigen provides a sufficient costimulatory signal for antigen-specific and MHC-restricted T cell activation. J. Immunol. 149:3802-3808.

10. Natesan, M., Razi-Wolf, Z., and Reiser, H. 1996. Costimulation of IL-4 production by murine $\mathrm{B} 7-1$ and $\mathrm{B} 7-2$ molecules. J. Immunol. 156:2783-2791.

11. Corry, R.J., Winn, H.J., and Russell, P.S. 1973. Primarily vascularized allografts of hearts in mice. The role of $\mathrm{H}-2 \mathrm{D}, \mathrm{H}-2 \mathrm{~K}$, and non-H-2 antigens in rejection. Transplantation. 16:343-350.

12. Waterhouse, P., et al. 1995. Lymphoproliferative disorders with early lethality in mice deficient in CTLA-4. Science. 270:985-988.

13. Chambers, C.A., Cado, D., Truong, T., and Allison, J.P. 1997. Thymocyte development is normal in CTLA-4-deficient mice. Proc. Natl. Acad. Sci. USA. 94:9296-9301.

14. Fraser, J.D., Irving, B.A., Crabtree, G.R., and Weiss, A. 1991. Regulation of interleukin-2 gene enhancer activity by the $\mathrm{T}$ cell accessory molecule CD28. Science. 251:313-316.

15. Rulifson, I.C., Sperling, A.I., Fields, P.E., Fitch, F.W., and Bluestone, J.A. 1997. CD28 costimulation promotes the production of Th2 cytokines. J. Immunol. 158:658-665.

16. Judge, T.A., et al. 1999. The role of CD80, CD86, and CTLA4 in alloimmune responses and the induction of long-term allograft survival. $J$. Immunol. 162:1947-1951.

17. Shahinian, A., et al. 1993. Differential T cell costimulatory requirements in CD28-deficient mice. Science. 261:609-612.

18. Freeman, G.J., et al. 2000. Engagement of the PD-1 immunoinhibitory receptor by a novel B7 family member leads to negative regulation of lymphocyte activation. J. Exp. Med. 192:1027-1034.

19. Green, J.M., et al. 1994. Absence of B7-dependent responses in CD28 deficient mice. Immunity. 1:501-508.

20. Wu, Y., Guo, Y., Huang, A., Zheng, P., and Liu, Y. 1997. CTLA4-B7 interaction is sufficient to costimulate T cell clonal expansion. J. Exp. Med. 185:1327-1335.

21. Abbas, A.K., and Sharpe, A.H. 1999. T-cell stimulation: an abundance of B7s. Nat. Med. 5:1345-1346.

22. Hutloff, A., et al. 1999. ICOS is an inducible T-cell co-stimulator structurally and functionally related to CD28. Nature. 397:263-266.

23. Mueller, D.L. 2000. T cells: a proliferation of costimulatory molecules. Curr. Biol. 10:R227-R230.

24. Brodie, D., et al. 2000. LICOS, a primordial costimulatory ligand? Curr. Biol. 10:333-336. 